\title{
Aplicación de un nuevo modelo metodológico en la docencia del Derecho Administrativo
}

\section{Application of a new methodological model in the teaching of Administrative Law}

JeRónimo CASTILLA GUERRA

Departamento de Derecho Administrativo

Universidad de Sevilla

icguerra@us.es

ORCID https://orcid.org/0000-0002-1604-1048

DOI: http://dx.doi.org/10.12795/9788447231003.011

Pp.: 236-255 


\section{Contexto}

La docencia del Derecho, al igual que otras disciplinas, se halla en la actualidad inmersa en un proceso de profunda revisión de sus paradigmas más clásicos. La docencia en torno a clases magistrales o fundamentada en el método del caso va cediendo espacio en favor de un modelo de corte más innovador, en el marco de las nuevas corrientes en educación y didáctica, con experiencias interesantes en el ámbito jurídico (Cubero, 2009; López, 2018; Soriano, 2019) en el que el alumnado se sitúa en el centro mismo de proceso.

En el presente trabajo se aborda, precisamente, el diseño, desarrollo y evaluación de un Ciclo de Mejora en el Aula (en adelante CIMA) en el área de Derecho Administrativo, en este caso en la asignatura "Introducción al Derecho del Turismo" del Grado de Turismo, impartido en el primer cuatrimestre del curso. Se trata de una materia obligatoria y consta de 6 créditos ECTS, que se traducen en 60 horas presenciales de clases teórico-prácticas (a razón de cuatro horas por semana divididas en dos días). El grupo no es excesivamente numeroso (60), lo cual nos permite llevar a cabo fácilmente actividades en grupos de trabajo que, en este caso, se han hecho coincidir con los tres grupos presenciales en los que fue dividido el grupo. El ciclo abarcó un total de 6 horas, es decir, 3 sesiones de 2 horas en el horario habitual de la asignatura: miércoles de 16 a 18 y viernes de 17 a 19.

El objetivo de la asignatura es introducir al alumnado en los conceptos básicos del Derecho Administrativo, dar a conocer la organización básica de la Administración pública, particularmente en el campo del turismo, así como, estudiar sus formas de actuación.

Dado que los resultados han sido satisfactorios, nuestra intención es utilizar el modelo metodológico y las dinámicas de trabajo del CIMA desarrollado en el conjunto de la asignatura.

Ciclos de Mejora en el Aula (2020). Experiencias de Innovación Docente de la US Esta obra se distribuye con la licencia Creative Commons 


\section{Diseño del Ciclo de Mejora en el Aula}

De acuerdo con lo previsto en el Curso General de Docencia Universitaria del Programa FIDOP de la Universidad de Sevilla, el diseño de un ciclo de mejora parte de la construcción de un mapa de contenidos y problemas, la definición de un modelo metodológico docente, de una secuencia de actividades a realizar en el aula y de un cuestionario inicial y final, con el que poder explorar las ideas del alumnado que definen sus modelos mentales.

\section{Mapa de Contenidos}

Procedemos a plantear un mapa de contenidos, tal y como reflejamos en la Figura 1, en el que optamos por un modelo en el que entendemos el procedimiento administrativo, no como un elemento o unidad independiente, sino como parte de un iter lógico (ligados por flechas en nuestro gráfico), en el que integramos el propio procedimiento, el acto administrativo o resolución subsiguiente y los eventuales recursos administrativos que pueden recaer sobre el mismo (todos en azul). Todos ellos siempre desplegados bajo al amparo del Ordenamiento jurídico que los regula y que trata de conciliar los intereses públicos y privados en juego (representados en amarillo).

Este supuesto permitirá al alumnado trabajar, sobre la base de un problema real, tanto el contenido conceptual, como el más actitudinal o ético, relacionado fundamentalmente con los valores en juego en el procedimiento, reflejados en el recuadro amarillo en la figura 1, y, finalmente, aquellos contenidos procedimentales que tienen una innegable implicación práctica y que hemos enmarcado en un recuadro verde.

Ciclos de Mejora en el Aula (2020). Experiencias de Innovación Docente de la US Esta obra se distribuye con la licencia Creative Commons 


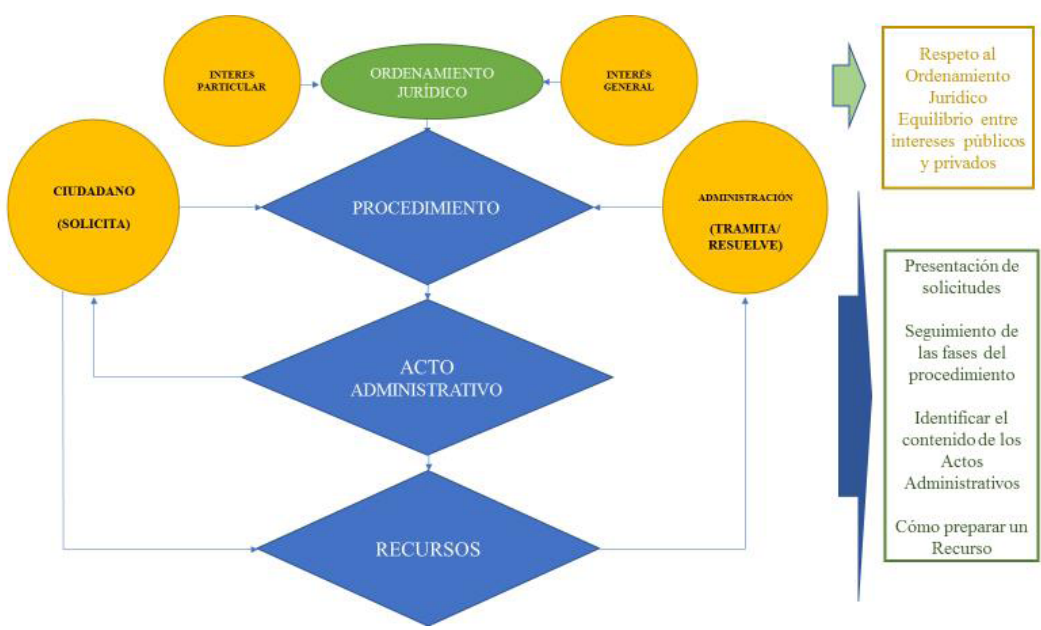

Figura 1. Mapa de Contenidos

\section{Cuestionario inicial-final}

Con el doble objetivo de poder valorar los conocimientos previos de los que parte el alumnado y de que tomen plena conciencia de cuál es su propio punto de partida, se les administró un formulario con varias preguntas que coinciden con los elementos clave del mapa de contenidos y el modelo metodológico propuesto en la figura 2.

Los resultados han sido determinantes, tanto a la hora de abordar el desarrollo de las actividades que se van a proponer para el aprendizaje en función de los problemas detectados, como para valorar, al final del proceso, el grado de aprendizaje del alumnado.

El cuestionario se planteó en torno a un supuesto real:

Vas a solicitar una licencia de apertura ante el Ayuntamiento para abrir un restaurante en tu barrio, por lo que debes estar familiarizado con los Procedimientos Administrativos. Para ello será necesario que puedas contestar una serie de cuestiones:

Ciclos de Mejora en el Aula (2020). Experiencias de Innovación Docente de la US Esta obra se distribuye con la licencia Creative Commons 
1. ¿Sabes para qué sirve y en qué consiste el procedimiento administrativo?

2. ¿Cómo deberían las nuevas tecnologías facilitar los trámites en los procedimientos administrativos?

3. ¿Cómo crees que puede resolver la Administración tu solicitud? ¿Qué contenido debería tener su respuesta?

\section{Modelo metodológico}

Se diseñó un modelo metodológico de corte investigativo (figura 2), de manera que el alumnado, situado en el centro del proceso, pueda adquirir el conocimiento en la materia mediante la propia indagación, apoyada en las actividades de contraste propuestas por el docente.


Figura 2. Modelo metodológico

El objetivo ha sido integrar su experiencia cotidiana, en torno a la obtención de una licencia de apertura de un negocio, como punto de partida para irlo aproximando a un análisis más jurídico relacionado con el Procedimiento Administrativo.

Ciclos de Mejora en el Aula (2020). Experiencias de Innovación Docente de la US Esta obra se distribuye con la licencia Creative Commons 
Para ello, hemos partido de los conocimientos previos que tienen sobre los problemas que se les suscitan desde la presentación de una solicitud en una Administración Pública hasta que, finalmente, surge el acto administrativo con el que se resuelve la cuestión.

Posteriormente, se les ha confrontado con la regulación jurídica subyacente, de manera que sean ellas y ellos los que, paulatinamente, vayan construyendo su propio conocimiento encajando todas las piezas a través de su experiencia en el aula, analizando su propio rol, no ya como ciudadano, sino como futuro profesional que deberá conocer las reglas del juego que rigen la interacción con las Administraciones.

\section{Secuencia de actividades}

A continuación, se describen las actividades realizadas en las tres sesiones en las que se aplica el CIMA, de acuerdo con el modelo y cuestiones señaladas.

Tabla 1. Secuencia de actividades del ciclo de mejora en el aula.

\begin{tabular}{|c|l|c|}
\hline ACTIVIDAD & DESCRIPCIÓN & Tiempo \\
\hline $\begin{array}{c}\text { N.o 0 } \\
\begin{array}{c}\text { Cuestionario } \\
\text { inicial }\end{array}\end{array}$ & $\begin{array}{l}\text { Previamente al inicio de estas sesiones } \\
\text { hemos pasado a través de la Plataforma } \\
\text { Virtual una encuesta con tres cuestiones } \\
\text { básicas sobre el tema a tratar en este CIMA. }\end{array}$ & \\
\hline \multicolumn{1}{|c|}{ SESIÓN 1 } \\
\hline $\begin{array}{c}\text { N.o 1 } \\
\text { Problema } \\
\text { inicial }\end{array}$ & $\begin{array}{l}\text { Plantear en clase la primera de las } \\
\text { cuestiones: ¿Sabes para qué sirve y en qué } \\
\text { consiste el procedimiento administrativo? } \\
\text { Abrimos un turno de palabra en el que } \\
\text { se les anima a expresar con libertad sus } \\
\text { ideas respecto a la pregunta propuesta }\end{array}$ & \\
\hline
\end{tabular}

Ciclos de Mejora en el Aula (2020). Experiencias de Innovación Docente de la US Esta obra se distribuye con la licencia Creative Commons 


\begin{tabular}{|c|c|c|}
\hline $\begin{array}{l}\text { N. o2 } \\
\text { Vídeo }\end{array}$ & $\begin{array}{l}\text { Para centrar la cuestión vemos un vídeo } \\
\text { introductorio, elaborado por juristas, sobre } \\
\text { el Procedimiento Administrativo en el que } \\
\text { se ponen de manifiesto sus aspectos más } \\
\text { relevantes. }\end{array}$ & $15^{\circ}$ \\
\hline $\begin{array}{l}\text { N. } 03 \\
\text { Debate en } \\
\text { clase }\end{array}$ & $\begin{array}{l}\text { Con los nuevos contenidos, abrimos un } \\
\text { nuevo turno de debate en el que se espera } \\
\text { que puedan introducir nuevos datos a la } \\
\text { luz del vídeo visionado }\end{array}$ & $15^{-}$ \\
\hline $\begin{array}{l}\text { N.o } 4 \\
\text { Revisión de } \\
\text { la normativa } \\
\text { vigente }\end{array}$ & $\begin{array}{l}\text { Partiendo de los inputs ofrecidos por } \\
\text { las alumnas y los alumnos, se presentan } \\
\text { por parte del profesor los fundamentos } \\
\text { teórico-jurídicos sobre la cuestión: Qué } \\
\text { dice la Constitución y la LPAC }\end{array}$ & $15^{\circ}$ \\
\hline $\begin{array}{l}\text { N.o } 5 \\
\text { Intervención } \\
\text { final del } \\
\text { alumnado }\end{array}$ & $\begin{array}{l}\text { Una vez presentado todo el contenido } \\
\text { conceptual intervienen nuevamente } \\
\text { resumiendo los aspectos clave del tema } \\
\text { tratado y se toma nota en la pizarra. }\end{array}$ & $20^{\circ}$ \\
\hline $\begin{array}{c}\text { N.o } 6 \\
\text { Sintesis }\end{array}$ & $\begin{array}{l}\text { Con idea de organizar y asentar los } \\
\text { conocimientos, se repasan conceptos } \\
\text { puestos de manifiesto en la sesión. }\end{array}$ & $10^{-}$ \\
\hline $\begin{array}{c}\text { N.o } 7 \\
\text { Caso práctico }\end{array}$ & $\begin{array}{l}\text { Para hilar lo aprendido en esta primera } \\
\text { sesión con la segunda, se les explica } \\
\text { la actividad que se ha colgado en la } \\
\text { plataforma virtual: Deberán localizar en } \\
\text { la web del Ayuntamiento de Sevilla el } \\
\text { procedimiento para pedir licencias de } \\
\text { inicio de actividad. Deberán cumplimentar } \\
\text { loscampos básicos de la solicitudyverificar } \\
\text { si pueden presentarla electrónicamente. }\end{array}$ & $10^{\prime}$ \\
\hline
\end{tabular}

\begin{tabular}{|c|l|c|}
\hline \multicolumn{3}{|c|}{ SESIÓN 2 } \\
\hline ACTIVIDAD & DESCRIPCIÓN & Tiempo \\
\hline $\begin{array}{c}\text { N.o 8 } \\
\text { Saludo } \\
\text { estudiantes y } \\
\text { recapitulación }\end{array}$ & $\begin{array}{l}\text { Comienza la sesión con un pequeño repaso } \\
\text { por parte del profesor de los contenidos } \\
\text { impartidos anteriormente y se resuelven }\end{array}$ & \\
\hline $\begin{array}{l}\text { las cuestiones o dudas pendientes del } \\
\text { alumnado }\end{array}$ & \\
\hline
\end{tabular}

Ciclos de Mejora en el Aula (2020). Experiencias de Innovación Docente de la US Esta obra se distribuye con la licencia Creative Commons Reconocimiento-NoComercial-SinObraDerivada 4.0 Internacional (CC BY-NC-ND 4.0.) 


\begin{tabular}{|c|c|c|}
\hline $\begin{array}{l}\text { № } 9 \\
\text { Problema } \\
\text { inicial }\end{array}$ & $\begin{array}{l}\text { Lanzamos la segunda cuestión: ¿Cómo } \\
\text { deberían las nuevas tecnologías facilitar } \\
\text { los trámites en los procedimientos } \\
\text { administrativos? Abrimos un nuevo debate } \\
\text { en que el que expresarán con libertad sus } \\
\text { ideas al respecto de la cuestión planteada. }\end{array}$ & 25 \\
\hline $\begin{array}{l}\text { N.o } 10 \\
\text { Vídeo }\end{array}$ & $\begin{array}{l}\text { Visualizamos vídeo sobre la Administración } \\
\text { electrónica elaborado por el Ministerio de } \\
\text { Administraciones Públicas. }\end{array}$ & 15 \\
\hline $\begin{array}{l}\text { № } 11 \\
\text { Resolución del } \\
\text { caso práctico }\end{array}$ & $\begin{array}{l}\text { Las y los estudiantes presentan la } \\
\text { información encontrada en casa fruto de } \\
\text { su investigación sobre un procedimiento } \\
\text { no sometido a la tramitación electrónica }\end{array}$ & 15 \\
\hline $\begin{array}{l}\text { N.o } 12 \\
\text { Revisión de } \\
\text { la normativa } \\
\text { aplicable }\end{array}$ & $\begin{array}{l}\text { Intervención del profesor en la que, } \\
\text { partiendo de las ideas de las alumnas } \\
\text { y los alumnos, se muestra el marco } \\
\text { jurídico relevante y las peculiaridades } \\
\text { de la administración electrónica en los } \\
\text { procedimientos administrativos. }\end{array}$ & 20 \\
\hline $\begin{array}{l}\text { N.o } 13 \\
\text { Sintesis }\end{array}$ & $\begin{array}{l}\text { Intervención individual del alumnado con } \\
\text { la que fijan las ideas sobre la materia } \\
\text { tratada en la sesión y se toma nota en la } \\
\text { pizarra }\end{array}$ & 10 \\
\hline $\begin{array}{c}\text { N.o } 14 \\
\text { Caso práctico }\end{array}$ & $\begin{array}{l}\text { Se les explica la actividad práctica } \\
\text { subida a la plataforma: Se les propone } \\
\text { un caso práctico real en el que el órgano } \\
\text { competente deniega el otorgamiento de } \\
\text { una licencia de apertura. Se les indica que } \\
\text { identifiquen los elementos que componen } \\
\text { el contenido de ésta. }\end{array}$ & 10 \\
\hline
\end{tabular}

\begin{tabular}{|c|l|c|}
\hline \multicolumn{3}{|c|}{ SESıón 3 } \\
\hline Actividad & Descripción & Tiempo \\
\hline $\begin{array}{c}\text { N.o 15 } \\
\text { Saludo } \\
\text { estudiantes y } \\
\text { recapitulación }\end{array}$ & $\begin{array}{l}\text { Comienza la sesión con un pequeño } \\
\text { repaso por el profesor de los contenidos } \\
\text { impartidos anteriormente y se resuelven } \\
\text { las cuestiones o dudas pendientes del } \\
\text { alumnado. }\end{array}$ & \\
\hline N.o 16 & $\begin{array}{l}\text { Lanzamos la última pregunta: ¿Cómo crees } \\
\text { que puede resolver la Administración tu } \\
\text { Pregunta inicial } \\
\text { solicitud? ¿Qué contenido debería tener } \\
\text { su respuesta? Se les da la palabra para } \\
\text { responder a esta cuestión. }\end{array}$ & \\
\hline
\end{tabular}

Ciclos de Mejora en el Aula (2020). Experiencias de Innovación Docente de la US Esta obra se distribuye con la licencia Creative Commons Reconocimiento-NoComercial-SinObraDerivada Internacional (CC BY-NC-ND 4.0.) 


\begin{tabular}{|c|l|c|}
\hline $\begin{array}{c}\text { N.o 17 } \\
\text { Resolución del } \\
\text { Caso Práctico }\end{array}$ & $\begin{array}{l}\text { Resolvemos en clase la actividad } \\
\text { práctica, analizando los elementos que } \\
\text { deben incorporar las resoluciones de la } \\
\text { Administración con las aportaciones del } \\
\text { alumnado. }\end{array}$ & $15^{\prime}$ \\
\hline $\begin{array}{c}\text { N.o 18 } \\
\text { Revisión de } \\
\text { la regulación } \\
\text { Jurídica }\end{array}$ & $\begin{array}{l}\text { Se pasa a exponer tanto la regulación } \\
\text { jurídica que rige la materia como la } \\
\text { jurisprudencia relevante. }\end{array}$ & $15^{\prime}$ \\
\hline $\begin{array}{c}\text { N.o 19 } \\
\text { Sintesis final } \\
\text { del alumnado }\end{array}$ & $\begin{array}{l}\text { Una vez presentados todos los } \\
\text { contenidos, intervienen individualmente } \\
\text { extrayendo las líneas maestras del tema } \\
\text { tratado en la sesión y se toma nota en la } \\
\text { pizarra de sus ideas. }\end{array}$ & $20^{-}$ \\
\hline $\begin{array}{c}\text { N.o 20 } \\
\text { Recapitulación }\end{array}$ & $\begin{array}{l}\text { A modo de cierre final, intervención del } \\
\text { profesor en la que se lleva a cabo una } \\
\text { recapitulación final de lo tratado en las } \\
\text { tres sesiones. }\end{array}$ & $15^{\prime}$ \\
\hline $\begin{array}{c}\text { N.o 21 } \\
\text { Cuestionario } \\
\text { final }\end{array}$ & $\begin{array}{l}\text { Para cerrar las sesiones se les informa } \\
\text { que se va a subir nuevamente a la } \\
\text { plataforma virtual la encuesta final para } \\
\text { poder extraer conclusiones sobre su } \\
\text { aprendizaje. }\end{array}$ & \\
\hline
\end{tabular}

\section{Diario de las sesiones}

A continuación, presentaré de manera sintética lo que podríamos llamar el relato resumido de las sesiones, es decir, cómo discurrieron junto a mis impresiones al respecto en cada momento.

\section{Sesión 0. Preparación}

Enfrentarnos a la elaboración de este CIMA resulta un importante desafío, en cuanto implica salir de nuestra zona de confort como docentes, de lo que hemos hecho siempre. Ahora bien, afrontar esta actividad en plena pandemia Covid supone un auténtico salto de trapecio sin red, que me hacía dudar del éxito de esta aventura.

Ciclos de Mejora en el Aula (2020). Experiencias de Innovación Docente de la US Esta obra se distribuye con la licencia Creative Commons 
Lo primero que hemos hecho, de acuerdo con nuestro modelo metodológico, es elaborar un formulario con tres cuestiones relacionadas con tres problemas esenciales de la asignatura y cuyos resultados han sido determinantes a la hora de establecer la secuencia de actividades.

\section{Sesión 1 (Duración 2h.)}

\section{Actividad 1. Pregunta inicial: ¿Sabes para qué sirve y en qué consiste el procedimiento administrativo?}

En nuestro modelo metodológico partimos de la formulación de una pregunta aparentemente simple con la que pretendemos verificar los conocimientos previos que las alumnas y los alumnos tienen sobre esta cuestión. Para esta primera aproximación aclaro e insisto en que no necesito argumentos jurídicos sino simplemente su opinión para poder fijar un punto de partida.

La actividad se puede decir que se desarrolló incluso mejor de lo que se tenía pensado. Aunque este nuevo planteamiento sorprende al alumnado, consigo captar su atención y eso resulta muy gratificante. La intervención de las alumnas y los alumnos en línea es evidente. El delegado del curso, que hace de moderador del foro, facilita sus intervenciones, si bien el grueso de las intervenciones parte de los asistentes a clase.

\section{Actividad 2. Vídeo}

Visualizamos un vídeo en el que se ponen de manifiesto las grandes cuestiones que suscita el Procedimiento Administrativo y en el que tienen la oportunidad de visualizar sus aspectos más relevantes desde el punto de vista jurídico. La visualización trascurre con normalidad.

\section{Actividad 3. Debate en la clase}

Con los nuevos datos que proporciona el vídeo iniciamos el debate en clase en el que pretendo que a su 
esquema inicial vayan incorporando estas nuevas ideas. En principio éste se desarrolla muy tímidamente, lo cual siempre genera dudas e inseguridades, pero, al insistir en que los argumentos a manejar no deben ser necesariamente jurídicos, el debate se anima. Me quedo más tranquilo al comprobar que el debate es fluido. Me limito a moderar el mismo y a fijar y aclarar ideas. Otra experiencia positiva.

\section{Actividad 4. Revisión de la normativa vigente}

Una vez que las alumnas y los alumnos están centrados en el tema, viene la parte más complicada y que, quizás, me despierta más inquietud ya que se trata de la única actividad en la que no tienen el protagonismo absoluto. Se trata de encajar las piezas más teóricas que les faltan en su puzle, los ladrillos técnico-jurídicos con los que sus argumentos cobran sentido. Partiendo de lo que al respecto dice la Constitución, completamos la visión de la regulación del Procedimiento Administrativo, para ello me valgo de una presentación breve en Power Point. Termina mi intervención y compruebo que aún siguen conectados. El éxito está en integrarla en el desarrollo lógico de esta sesión y en la brevedad de ésta.

\section{Actividad 5. Intervención final del alumnado}

Intervienen los estudiantes resumiendo las ideas claves de las que tomo nota en la pizarra. Si bien las intervenciones de los que asisten a clase no resultan problemáticas, la intervención del resto del grupo, que sigue la actividad online, no llega al nivel de lo deseable y trato en varias ocasiones de provocar más intervenciones de este sector. Tardamos más de lo esperado en tener un esquema completo en la pizarra.

\section{Actividad 6. Síntesis}

Tras la intervención previa de las alumnas y los alumnos, prácticamente no se hace necesaria la síntesis que Ciclos de Mejora en el Aula (2020). Experiencias de Innovación Docente de la US
Esta obra se distribuye con la licencia Creative Commons 
dura menos de lo previsto. Les indico que esta actividad en las sesiones siguientes no la hará el profesor.

\section{Actividad 7. Caso Práctico}

Para asentar lo aprendido y que tengan un contacto con procedimientos reales les doy instrucciones de localizar un procedimiento muy concreto, sobre la solicitud de una licencia de inicio de actividad en la web del Ayuntamiento, debiendo preparar la solicitud y averiguar dónde, cómo se inicia y cómo discurre el procedimiento. Desde el foro virtual recibo algunas consultas al respecto que aprovecho para aclarar a todo el grupo. La explicación del caso dura más de lo previsto, pero encaja en el tiempo de esta sesión.

Al salir de clase, lo primero que hice fue plasmar por escrito en la Enseñanza Virtual todas las indicaciones que les había dado.

\section{Sesión2 (Duración 2h.)}

\section{Actividad 8. Saludo y recapitulación}

Comienza la sesión con un pequeño repaso por parte del profesor de los contenidos impartidos anteriormente y se resuelven las cuestiones o dudas pendientes del alumnado. Pretendo intervenir lo menos posible pero esta actividad se demora más de lo previsto. Las alumnas y los alumnos asistentes, que en la sesión anterior estaban online, tratan de despejar las dudas que no se lanzaron on line.

\section{Actividad 9. Problema inicial}

Lanzamos la segunda cuestión prevista: ¿Cómo deberían las nuevas tecnologías facilitar los trámites en los procedimientos administrativos? Abrimos un nuevo debate en el que expresaron con libertad sus ideas al respecto de la cuestión planteada.

Ciclos de Mejora en el Aula (2020). Experiencias de Innovación Docente de la US Esta obra se distribuye con la licencia Creative Commons 
Pude comprobar con satisfacción que, en esta ocasión, se manejaron con soltura ciertos conocimientos que han surgido de la clase anterior. La nueva metodología parece que va dando algunos frutos. Además, las intervenciones online son algo más fluidas y trato de motivar directamente a las alumnas y los alumnos más activos de la sesión pasada para dinamizar más la clase.

Los resultados, en general, son algo más satisfactorios que en la sesión anterior.

\section{Actividad 10. Vídeo}

En esta ocasión, visualizamos un vídeo sobre la Administración electrónica, la e-Administración, elaborada por el Ministerio de Hacienda y Administraciones Públicas. Se trata de un vídeo en el que se ponen de manifiesto las ventajas que aporta la administración electrónica en la tramitación de los distintos procedimientos administrativos y en el que tienen la oportunidad de visualizar sus aspectos más relevantes. La visualización trascurre con normalidad.

\section{Actividad 11. Resolución caso práctico}

Pasamos a revisar los resultados del caso práctico. Me da la impresión de que los únicos que intervienen son los que asisten a clase, así que me dirijo al grupo que sigue las clases online y la sesión se convierte en algo más dinámica y participativa. Creo que el objetivo de que vean cómo se inician los procedimientos y sus distintas vías se cumple satisfactoriamente.

\section{Actividad 12. Revisión de la normativa aplicable}

Mi intervención como profesor se concentra en esta actividad en la que, aprovechando sus aportaciones previas, se presentan ordenadamente los contenidos jurídicos esenciales sobre las formas de iniciar el procedimiento y las distintas fases por la que discurre (apoyado en una breve presentación PPT) con el que pretendo apuntalar las ideas que ya han puesto previamente de manifiesto. Ciclos de Mejora en el Aula (2020). Experiencias de Innovación Docente de la US 


\section{Actividad 15. Sintesis}

Empiezo a pensar que el éxito o fracaso de las sesiones va a estar muy vinculado al resultado de esta actividad, en la que puedo ver con nitidez si realmente han comprendido todo lo analizado en las distintas actividades.

Las alumnas y los alumnos van interviniendo con cierta fluidez y vamos tomando nota en la pizarra de las ideas que van aportando individualmente. Entre todos trazan un mapa bastante completo de lo que pretendía ver en esta sesión. Puedo estar contento con el resultado.

\section{Sesión 3 (Duración 2h.)}

\section{Actividad 15. Saludo y recapitulación}

Preparamos los medios audiovisuales y el material de clases. Doy un breve repaso a los contenidos impartidos en la última clase y resuelvo alguna duda puntual. En esta ocasión estamos dentro del tiempo estimado.

\section{Actividad 16. Pregunta inicial: ¿Cómo crees que puede resolver la Administración tu solicitud? ¿Qué contenido debería tener su respuesta?}

Siguiendo nuestro modelo metodológico partimos nuevamente de la formulación de una pregunta, la tercera, para explorar los conocimientos previos con los que cuentan. Para esta primera aproximación aclaro e insisto en que no necesito necesariamente argumentos jurídicos sino simplemente su opinión.

En esta ocasión vuelvo a comprobar con satisfacción que manejan con corrección conceptos y expresiones planteados en sesiones previas. Podemos estar contentos con el resultado de esta actividad pese a las circunstancias actuales.

\section{Actividad 17. Resolución caso práctico}

Esta actividad se había planteado a modo de resumen general y conclusión lógica de los contenidos analizados 
en las sesiones previas y dejaría sobre la mesa las cuestiones relacionadas con el acto y los recursos administrativos que se tratarán en otras sesiones, de ahí su importancia.

Veo que la dinámica de las sesiones anteriores ha sentado las bases para que esta actividad transcurra con cierta soltura. Mis intervenciones se centran en buscar las opiniones del alumnado al que le cuesta intervenir, especialmente en la modalidad online.

La actividad discurre bien, aunque nos excedemos del tiempo previsto. No obstante, lo doy por bien empleado ya que van aflorando de manera natural cuestiones de gran interés.

\section{Actividad 18. Revisión de la regulación}

Esta actividad es casi una consecuencia inevitable de la anterior, ya que les va a facilitar las herramientas jurídicas con las que dar fundamento a todos sus argumentos. Nuevamente mi intervención como profesor se limita a presentar ordenadamente, y partiendo de las intervenciones previas de las alumnas y los alumnos, los contenidos jurídicos esenciales de las cuestiones tratadas. Al igual que en la sesión anterior me apoyo en una breve presentación PPT. La actividad trascurre en tiempo y forma.

\section{Actividad 19. Síntesis final del alumnado}

Intervienen ahora con más seguridad, aportando las principales líneas de síntesis del tema tratado y vamos tomando nota de estas en la pizarra. Nuevamente el resultado es bastante satisfactorio.

\section{Actividad 20. Recapitulación}

Intervengo finalmente para mostrarles el camino recorrido y hasta dónde hemos llegado en este viaje juntos a lo largo y ancho de estas tres últimas sesiones. Veo en sus caras de satisfacción (en la de los alumnos que asisten a clase) que han disfrutado de este nuevo estilo de aprendizaje.

Ciclos de Mejora en el Aula (2020). Experiencias de Innovación Docente de la US Esta obra se distribuye con la licencia Creative Commons 


\section{Actividad 21. Cuestionario final}

Por último, procedo a pasar nuevamente las encuestas iniciales a fin de valorar el resultado del proceso de aprendizaje. Será el momento de ver si la nueva metodología docente da sus frutos.

Por cerrar estas reflexiones, puedo señalar, a modo de conclusión, que la experiencia ha sido sumamente gratificante. Aún siendo consciente de que sigue siendo sólo una gota en la inmensidad del curso y de que aún queda mucho por aprender para la mejora de mi actividad docente, los cambios introducidos me han permitido conectar de manera más estrecha con el alumnado.

Además, al sentirse más protagonistas, abandonan su clásico papel de meros sujetos pasivos y cambian de manera natural su actitud en las clases, integrándose de manera orgánica en el proceso de aprendizaje.

\section{Evaluación del aprendizaje de los estudiantes}

Tal y como se ha precisado, tras las tres sesiones volvimos a pasar el cuestionario para evaluar los cambios en los modelos mentales del alumnado y los conocimientos conceptuales, actitudinales y procedimentales adquiridos durante las sesiones.

De su análisis extraemos las siguientes tres escaleras de aprendizajes, coincidiendo con las tres preguntas planteadas (figuras 1, 2 y 3). En función de las respuestas obtenidas hemos podido establecer cuatro modelos diferentes que identificamos gráficamente con cuatro escalones. Estos nos ofrecen una información muy valiosa a la hora de detectar los obstáculos más relevantes en el aprendizaje y nos han permitido situar a cada estudiante dentro de un nivel determinado.

Del resultado final en cada respuesta, destacado en color azul, podemos extraer una conclusión inmediata muy 
positiva, por cuanto la evolución en el aprendizaje nos ha llevado a situar al grueso del alumnado en los dos últimos escalones en cada una de las preguntas (90\%, 85\% y $78 \%$, respectivamente), experimentando, por tanto, una evolución en su aprendizaje muy satisfactoria.

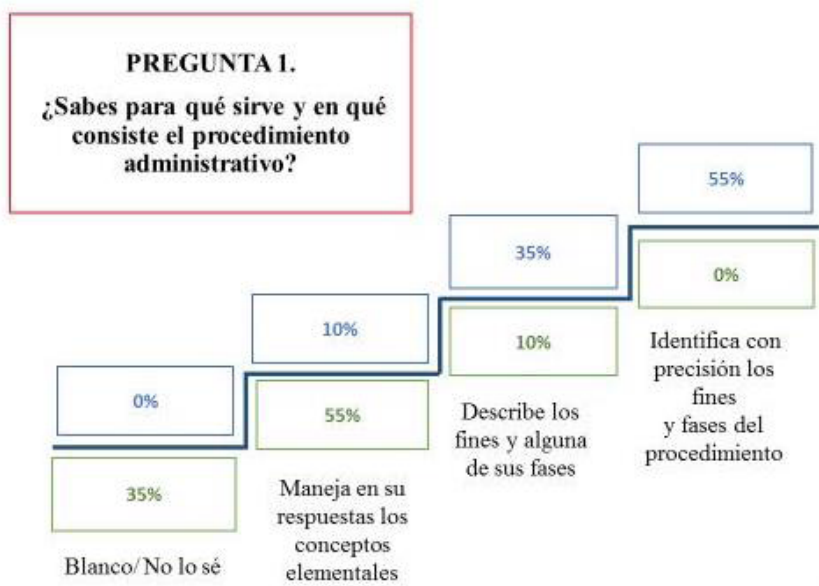

CUESTIONARIO FINAL

CUESTIONARIO INICIAL

Figura 3. Escalera 1

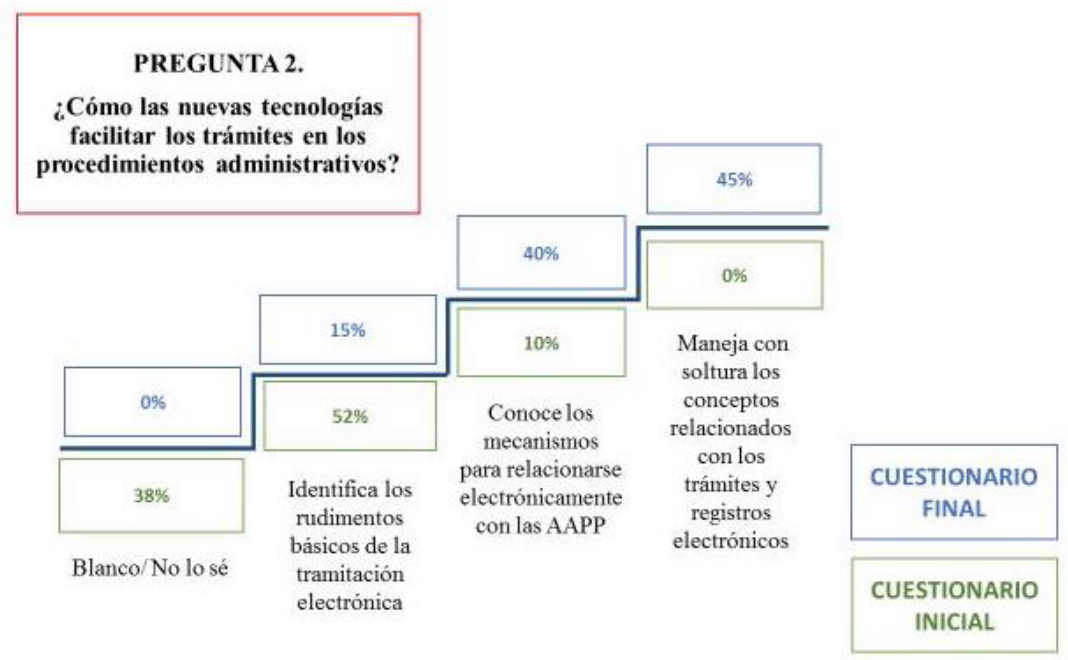

Figura 4. Escalera 2

Ciclos de Mejora en el Aula (2020). Experiencias de Innovación Docente de la US (c) Esta obra se distribuye con la licencia Creative Commons Reconocimiento-NoComercial-SinObraDerivada Internacional (CC BY-NC-ND 4.0.) 


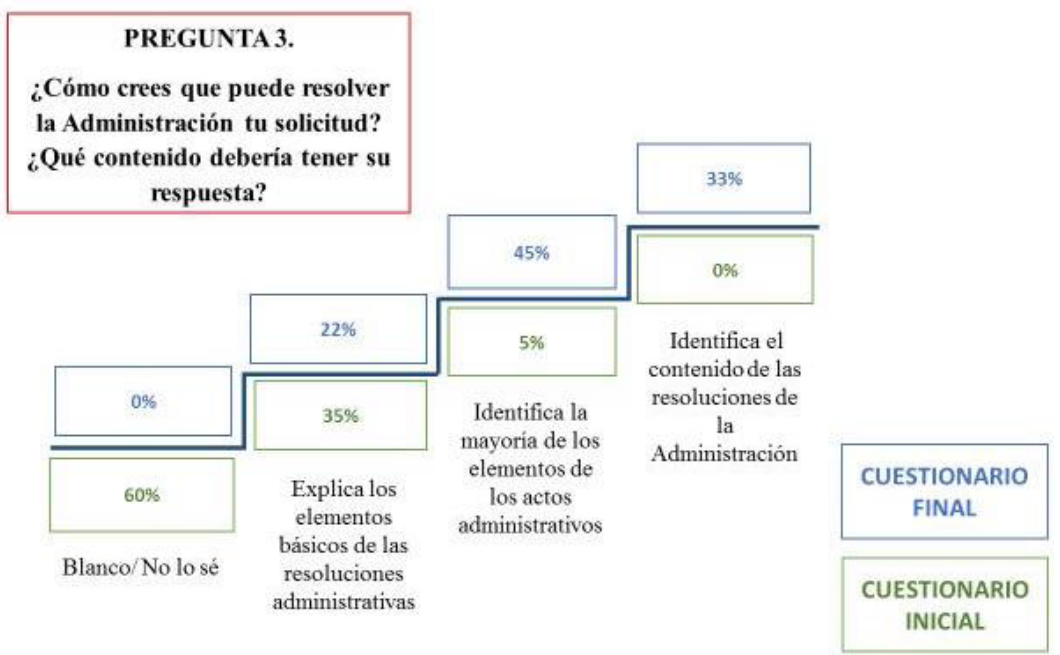

Figura 5. Escalera 3

\section{Evaluación del Ciclo de Mejora en el Aula}

\section{Aspectos de la experiencia que se pretenden incorporar a la práctica docente habitual}

La implementación del CIMA en la práctica docente ha sido especialmente gratificante. El nivel de participación del alumnado en las actividades propuestas y el nivel de aprendizaje obtenido ha superado con creces mis expectativas iniciales, máxime en unas circunstancias tan complicadas.

El hecho de desplazar el eje de la docencia hacia el propio alumnado ha sido fundamental en el éxito de esta experiencia, no solo a la hora de abordar el estudio como un proceso investigativo en el que poco a poco han ido descubriendo el contenido objeto de estudio, sino porque ha supuesto un nivel de compromiso mayor que ha facilitado su participación mayoritariamente activa. De igual modo, los comentarios expresados por el grupo sobre esta novedosa práctica docente han sido igualmente positivos.

Ciclos de Mejora en el Aula (2020). Experiencias de Innovación Docente de la US Esta obra se distribuye con la licencia Creative Commons 
La consecuencia lógica de esta valoración resulta evidente. Para lograr unos mejores resultados debiera implementarse este modelo metodológico en la docencia habitual de esta asignatura.

\section{Principios didácticos}

De toda la elaboración de este CIMA, he creído pertinente seleccionar aquellos principios didácticos que he considerado más relevantes y en los que se ha cimentado el modelo didáctico personal que he seguido.

En relación con los contenidos, partiremos de una visión integradora en nuestro mapa de contenidos en el que deben tener cabida conceptos, procedimientos y actitudes (Porlán 2017), siempre partiendo, no de meras formulaciones teóricas, sino de problemas reales de la disciplina. En este modelo, se entiende el conocimiento como un proceso que requiere de su compromiso (Bain, 2007) para que, mediante la formulación de problemas reales y desafiantes sean capaces de alcanzar sus propias conclusiones (Finkel, 2008).

La elaboración de un cuestionario con dichos problemas nos ofrecerá una información crucial a la hora de ajustar nuestro mapa de contenidos, así como para diseñar una adecuada secuencia de actividades que nos facilite uno de los grandes objetivos metodológicos de nuestro modelo, esto es, la involucración del alumnado en su propio proceso de aprendizaje, abandonando, de este modo, su clásico papel de mero consumidor pasivo (Porlán, 2017).

Por último y como colofón necesario a este modelo, se debe acometer la valoración final de los aprendizajes alcanzados partiendo de los mismos cuestionarios que, una vez terminadas las sesiones vuelven a responder, ofreciéndonos la información necesaria para analizar los cambios observados y que permitirán al docente reflexionar sobre los resultados en su diario y afrontar posibles mejoras en el próximo curso (Porlán, 2017). 
Palabras clave: Derecho Administrativo; Derecho del Turismo; docencia universitaria; experimentación docente universitaria.

Keywords: Administrative Law; Tourism Law; University teaching; University teaching experimentation.

\section{Referencias Bibliográficas}

Bain, K. (2007). Lo que hacen los mejores profesores universitarios. Valencia: Publicaciones Universidad de Valencia.

Cubero, A. (2009). El aprendizaje basado en problemas aplicado al Derecho Tributario. El alumno asume el rol de asesor fiscal especializado. En IX Jornada sobre Aprendizaje Cooperativo y II Jornada sobre Innovación Docente, Almería, 9-10 julio.

Finkel, D. (2008). Dar clases con la boca cerrada. Valencia: Publicaciones Universidad de Valencia.

López, A. (2018). Somos juristas. Un taller conceptual. Jornadas de Formación e Innovación Docente del Profesorado. No 1. I Jornadas FIDOP Universidad de Sevilla, 374-384.

Porlán, R. (Coord.) (2017). Enseñanza universitaria. Cómo mejorarla. Madrid: Morata

Soriano, J. (2019). El aprendizaje a través de la simulación: Modelo de la UE en la asignatura "Instituciones Jurídicas de la Unión Europea”. En E. Navarro-Medina y R. Porlán (Coord.), Ciclos de mejora en el aula. Año 2019. Experiencias de innovación docente de la Universidad de Sevilla (pp. 2631-2651). Sevilla: Editorial de la Universidad de Sevilla

Ciclos de Mejora en el Aula (2020). Experiencias de Innovación Docente de la US Esta obra se distribuye con la licencia Creative Commons 